\title{
由猪去氧胆酸合成异熊去氧胆酸的新方法
}

\author{
刘双汪钢强刘张坤汪秋安* \\ (湖南大学化学化工学院 长沙 410082)
}

\begin{abstract}
摘要 研究了异熊去氧胆酸的合成新方法. 以猪去氧胆酸为原料, 通过 9 步反应(总产率为 $25 \%$ ), 高效地合成了异熊去 氧胆酸. 合成路线中的关键中间体 4 是由化合物 3 经改进的烯丙位氧化以较高产率获得. 合成反应的关键步骤分别是 化合物 5 在 Mistunobu 反应条件下的构型转化和 $\alpha, \beta$-不饱和羰基化合物 $\mathbf{6}$ 发生的立体选择性 Luche 还原. 所有合成化合 物的结构已通过 ${ }^{1} \mathrm{H} N \mathrm{NM},{ }^{13} \mathrm{C} \mathrm{NMR}, \mathrm{MS}$ 和 IR 等波谱方法进行了确认. 该合成途径原料易得、总产率较高、后处理操 作简便，具有较高的应用价值.
\end{abstract}

关键词＼cjkstart异熊去氧胆酸; 猪去氧胆酸; Mitsunobu 构型反转; Luche 还原; 合成

\section{A Novel Synthetic Approach of Alloursodeoxycholic Acid from Hydeoxycholic Acid}

\author{
Liu, Shuang Wang, Gangqiang Liu, Zhangkun Wang, Qiuan* \\ (College of Chemistry and Chemical Engineering, Hunan University, Changsha 410082)
}

\begin{abstract}
A novel and highly efficient synthetic approach of alloursodeoxycholic acid, starting from hydeoxycholic acid in 9 steps with overall yield $25 \%$, was reported. The key intermediate 4 was prepared in good yield by improved allylic oxidation of 3. The critical steps to stereoselectively inverse the configuration of $\mathbf{5}$ and $\alpha, \beta$-unsaturated carbonyl reduction of $\mathbf{6}$ were realized under Mistunobu coditions and Luche reduction respectively. The structures of all synthetic compounds were confirmed by ${ }^{1} \mathrm{H}$ NMR, ${ }^{13} \mathrm{C}$ NMR, MS and IR spectra. The synthetic approach has the advantages of easy availability of starting materials, highly stereoselective, easily work-up and good overall yield, so it has considerable practical value.
\end{abstract}

Keywords alloursodeoxycholic acid; hydeoxycholic acid; Mistunobu configuration inversion; Luche reduction; synthesis

异熊去氧胆酸 (Alloursodeoxycholic acid), 又名 $3 \alpha, 7 \beta$-二羟基- $5 \alpha$-胆烷酸, 是熊去氧胆酸(Ursodeoxycholic acid, 又名 $3 \alpha, 7 \beta$-二羟基- $5 \beta$-胆烷酸)的差向异构体 ${ }^{[1]}$. 熊去氧胆酸系珍贵药物熊胆的有效成分, 具有清热、平 肝、明目等功效. 临床上用于治疗各种胆道疾病、急慢 性肝炎、肝硬化及心血管疾病, 尤其对胆固醇型胆结石 有独特疗效 ${ }^{[2]}$. 异熊去氧胆酸以及它的系列衍生物是存 在于哺乳动物包括人体胆囊中的微量成分, 作为人体胆 固醇代谢为胆酸过程中的一个重要中间体 ${ }^{[3]}$, 具有重要 的生理功能. 生化分析在检测人体胆固醇代谢指标时, 需采用高纯度异熊去氧胆酸作为标准物质, 同时异熊去 氧胆酸的药学价值也尚待研发. 因此有必要对异熊去氧 胆酸的合成新方法进行研究.
自 20 世纪 50 年代起, 人们在试图用人工方法合成 熊去氧胆酸, 也曾对异熊去氧胆酸的合成进行了研究. Goto 和 Iida 等 ${ }^{[4,1]}$ 分别提出了从 $3 \alpha$-乙氧碳酰-6-澳-7,12二酮- $5 \beta$-胆烷酸甲酯和 $4 \beta$-溴- $7 \alpha$-甲酰氧基- 3 -酮 $-5 \beta$-胆烷 酸合成异熊去氧胆酸的路线, 他们采用的合成方法原料 难得, 反应步骤多, 且反应中采用碱金属钠在醇中还原, 反应十分剧烈，或使用过氧化钾等危险品，因此反应操 作十分不便. 猪去氧胆酸(Hydeoxycholic acid)是从猪胆 汁中提出来的天然甾体化合物, 其来源丰富, 价廉易得. 以含量较高且来源丰富的猪去氧胆酸为原料半合成异 熊去氧胆酸, 具有原料易得、合成步骤少、生产成本低 和环境友好等优点, 对药物和生化产品开发具有重要意 义.

\footnotetext{
*E-mail:wangqa@hnu.edu.cn

Received April 20, 2013; revised June 25, 2013; published online July 3, 2013.

Project supported by the Personal Training Funds in National Basic Science of China (No. J1210040/J0104) and the Hunan Provincial Innovation Foundation for Postgraduate (No. CX2012B160).

国家基础科学人才培养基金(No. J1210040/J0104)和湖南省研究生科研创新(No. CX2012B160)资助项目.
} 
我们以来源丰富的猪去氧胆酸为原料, 经过酯化、 消除反应、烯丙位氧化、Mistunobu 构型转化、 Luche 还原、催化氢化和酯水解等反应步骤, 完成了异熊去氧 胆酸的合成, 对每一步反应的反应条件进行了优化, 对 产物用核磁共振(NMR)、质谱(MS)和红外光谱(IR)等波 谱方法进行了结构确证. 合成路线见 Scheme 1.

\section{1 实验部分}

\section{1 仪器与试剂}

熔点在 XRC-1 型显微熔点仪上测定, 温度未加校 正; NMR 用 Bruker Am-400 型核磁共振仪测定(溶剂 $\mathrm{CDCl}_{3}$ 或 DMSO- $d_{6}, \mathrm{TMS}$ 为内标); 质谱用 Agilent 1100 液-质联用仪或 ZAB-HS 型仪测定记录; 红外光谱用 FD-5DX 仪(KBr 压片)测定; 柱层析用硅胶(青岛海洋化 工厂产品); 所用试剂和溶剂为化学纯或分析纯; 要求 无水的溶剂均经除水和重蒸处理.

猪去氧胆酸甲酯(1), $3 \alpha, 6 \alpha$-双对甲苯磺酰氧基胆烷 酸甲酯(2), $3 \beta$-乙酰基- $\Delta^{5}$-胆烷酸甲酯(3)按文献合成 ${ }^{[5,6]}$.

\section{$1.23 \beta$-乙酰氧基-7-酮基- $\Delta^{5}$-胆烷酸甲酯(4)的合成}

$0.5 \mathrm{~g}(5 \mathrm{mmol})$ 的 $\mathrm{CrO}_{3}$ (经过 $\mathrm{P}_{2} \mathrm{O}_{5}$ 干燥过) 溶解于 3 $\mathrm{mL}$ 吡啶的 $30 \mathrm{~mL} \mathrm{CH}_{2} \mathrm{Cl}_{2}$ 中, 搅拌 $10 \mathrm{~min}$ 后, 加入 30.5 $\mathrm{g}(1.2 \mathrm{mmol})$ 的 $10 \mathrm{~mL} \mathrm{CH} \mathrm{Cl}_{2}$ 溶液，再加入 $0.5 \mathrm{~mL} \mathrm{70 \%}$ 过氧叔丁醇(TBHP), 室温搅拌 $12 \mathrm{~h}$, 滤去固体, 滤液用 乙酸乙酯萃取 $(15 \mathrm{~mL} \times 3)$ 有机相用 $10 \%$ 的 $\mathrm{HCl}$ 溶液，饱 和 $\mathrm{NaHCO}_{3}$ 溶液洗涤, 无水 $\mathrm{Na}_{2} \mathrm{SO}_{4}$ 干燥, 浓缩得粗品. 快速硅胶柱层析 [洗脱剂: $V$ (石油醚) : $V$ (乙酸乙酯 $)=$ 9：1]得白色固体 $40.26 \mathrm{~g}$, 产率为 80\%. m.p. 168 169 ${ }^{\circ} \mathrm{C}$ (文献值 ${ }^{[6]}$ : m.p. $167 \sim 169{ }^{\circ} \mathrm{C}$ ); ${ }^{1} \mathrm{H}$ NMR (400 MHz, $\left.\mathrm{CDCl}_{3}\right) \delta: 0.68\left(\mathrm{~s}, 3 \mathrm{H}, 18-\mathrm{CH}_{3}\right), 0.95(\mathrm{~d}, J=6.4 \mathrm{~Hz}, 3 \mathrm{H}$, $\left.21-\mathrm{CH}_{3}\right), 1.05 \sim 2.60(\mathrm{~m}, 23 \mathrm{H}), 1.22\left(\mathrm{~s}, 3 \mathrm{H}, 19-\mathrm{CH}_{3}\right), 2.13$ (s, 3H, $\left.3 \beta-\mathrm{OCOCH}_{3}\right), 3.69$ (s, 3H, $\left.\mathrm{COOCH}_{3}\right), 4.71$ (brm, $1 \mathrm{H}, 3 \alpha-\mathrm{H}), 5.70$ (brs, 1H, 6-H); IR $v_{\max }: 1665,1740(\mathrm{C}=$ O) $\mathrm{cm}^{-1}$; HR-MS (EI) calcd for $\mathrm{C}_{27} \mathrm{H}_{40} \mathrm{O}_{5}$ : 444.2876, found 444.2871.

\section{$1.33 \beta$-差货基-7-酮基- $\Delta^{5}$-胆烷酸甲酯(5)的合成}

化合物 $40.444 \mathrm{~g}$ (1 mmol) 溶解于 $2 \mathrm{~mL}$ 的甲醇中, 再加入 $5 \mathrm{~mL} \mathrm{KHCO}_{3} / \mathrm{K}_{2} \mathrm{CO}_{3}\left(\mathrm{KHCO}_{3}\right.$ 和 $\mathrm{K}_{2} \mathrm{CO}_{3}$ 都为 0.5 $\mathrm{mol} / \mathrm{L})$ 溶液, 室温反应 $3 \mathrm{~h}$, 乙酸乙酯萃取 $(15 \mathrm{~mL} \times 3)$, 有机相用饱和 $\mathrm{NaCl}$ 溶液洗涤, 无水 $\mathrm{Na}_{2} \mathrm{SO}_{4}$ 干燥, 浓缩 得粗品. 快速硅胶柱层析[洗脱剂: $V$ (石油醚)： $V$ (乙酸 乙酯 $)=8: 2$ ]得白色固体 $50.39 \mathrm{~g}$, 产率为 97\%. m.p. $140 \sim 141{ }^{\circ} \mathrm{C}$ (文献值 ${ }^{[7]}$ : m.p. $\left.141 \sim 142{ }^{\circ} \mathrm{C}\right) ;{ }^{1} \mathrm{H}$ NMR $(400$

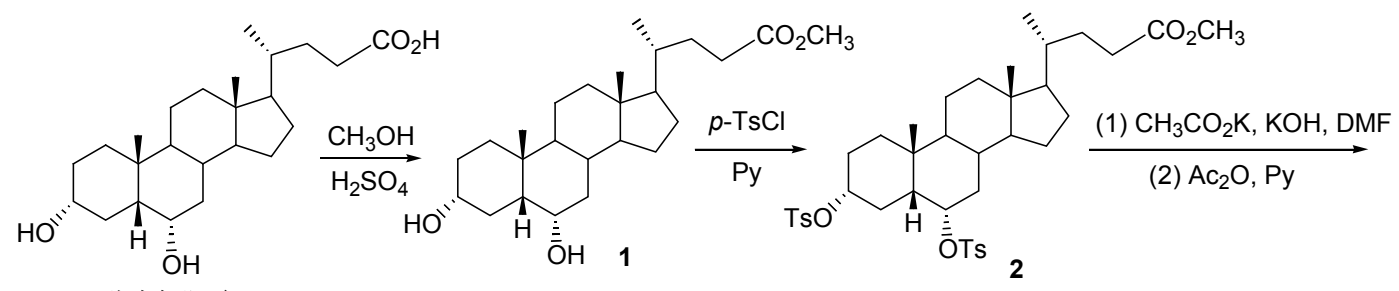

猪去氧胆酸<smiles>CC(=O)OC(=O)CC[C@@H](C)C1CCC2C3CC=C4CC(OC(C)=O)CC[C@@]4(C)C3CCC21C</smiles>

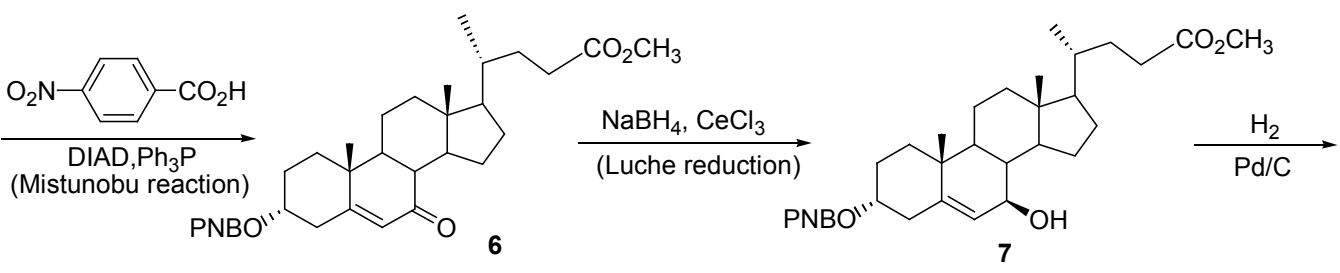

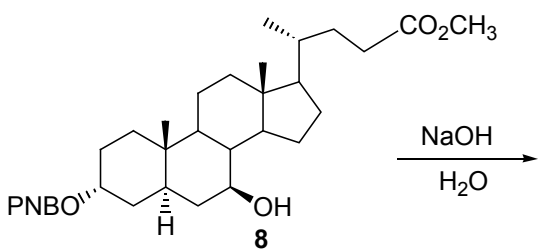<smiles>C[C@H](CCC(=O)O)C1CCC2C3C(O)C[C@@H]4C[C@@H](O)CC[C@]4(C)C3CC[C@@]21C</smiles>

Scheme 1 
$\left.\mathrm{MHz}, \mathrm{CDCl}_{3}\right) \delta: 0.69\left(\mathrm{~s}, 3 \mathrm{H}, 18-\mathrm{CH}_{3}\right), 0.94(\mathrm{~d}, J=6.2 \mathrm{~Hz}$, $\left.3 \mathrm{H}, 21-\mathrm{CH}_{3}\right), 1.05 \sim 2.57(\mathrm{~m}, 23 \mathrm{H}), 1.22\left(\mathrm{~s}, 3 \mathrm{H}, 19-\mathrm{CH}_{3}\right)$, $3.60 \sim 3.63$ (brm, 1H, $3 \alpha-\mathrm{H}), 3.67$ (s, $\left.3 \mathrm{H}, \mathrm{COOCH}_{3}\right), 5.67$ (brs, 1H, 6-H); ${ }^{13} \mathrm{CNMR}\left(100 \mathrm{MHz}, \mathrm{DMSO}-d_{6}\right) \delta: 201.4$ (C-7), 174.2 (C-24), 167.1 (C-5), 125.2 (C-6), 69.5 (C-14), 55.7 (C-3), 54.3 (C-17), 52.7 (C-25), 51.6 (C-9), 20.3 (C-10), 19.6 (C-21), 12.2 (C-19, C-18); IR $v_{\max }: 1672$, $1720(\mathrm{C}=\mathrm{O}), 3475(\mathrm{O}-\mathrm{H}) \mathrm{cm}^{-1}$. HRMS (EI) calcd for $\mathrm{C}_{25} \mathrm{H}_{38} \mathrm{O}_{4}$ : 402.2760, found 402.2768.

\section{$1.43 \alpha$-对硝基苯甲酰基-7-酮基- $\Delta^{5}$-胆烷酸甲酯(6)的 合成}

在 $250 \mathrm{~mL}$ 的圆底烧瓶中加入用 $75 \mathrm{~mL}$ 无水 $\mathrm{THF}$ 溶 解的化合物 $5(28.7 \mathrm{mmol})$ 、三苯基膦 $(57.4 \mathrm{mmol})$ 和对硝 基苯甲酸 $(57.4 \mathrm{mmol})$, 用恒压滴液漏斗慢慢滴加经甲苯 稀释的偶氮二甲酸二异丙酯(DIAD) $(57.4 \mathrm{mmol})$ $[V(\mathrm{DIAD}): V$ (甲苯 $)=2: 5$ ], 在 $\mathrm{N}_{2}$ 条件下室温傥拌 24 h. 向反应中加入到 $94.8 \mathrm{~mL} \mathrm{NaOH}(1 \mathrm{~mol} / \mathrm{L})$ 溶液室温摚 拌 $1 \mathrm{~h}$, 减压除去大部分的有机溶剂, 再向体系中加入 $50 \mathrm{~mL}$ 水, 乙酸乙酯萃取 $(50 \mathrm{~mL} \times 3)$, 有机相用饱和 $\mathrm{NaCl}$ 溶液洗涤, 无水 $\mathrm{Na}_{2} \mathrm{SO}_{4}$ 干燥, 浓缩得粗品. 快速硅 胶柱层析[洗脱剂: $V$ (石油醚) $: V$ (乙酸乙酯 $)=9 ： 1$ ] 得白 色固体 $613.6 \mathrm{~g}$, 产率为 $86 \%$. m.p. $157 \sim 160{ }^{\circ} \mathrm{C} ;{ }^{1} \mathrm{H}$ NMR (400 MHz, $\left.\mathrm{CDCl}_{3}\right) \delta: 0.64\left(\mathrm{~s}, 3 \mathrm{H}, 18-\mathrm{CH}_{3}\right), 0.90(\mathrm{~d}$, $\left.J=6.2 \mathrm{~Hz}, 3 \mathrm{H}, 21-\mathrm{CH}_{3}\right), 0.91 \sim 2.70(\mathrm{~m}, 23 \mathrm{H}), 1.22(\mathrm{~s}$, $3 \mathrm{H}, 19-\mathrm{CH}_{3}$ ), 3.60 (s, $3 \mathrm{H}, \mathrm{COOCH}_{3}$ ), 5.42 (brs, $1 \mathrm{H}, 3-\mathrm{H}$ ), 5.72 (brs, $1 \mathrm{H}, 6-\mathrm{H}), 8.12$ (d, $J=8.0 \mathrm{~Hz}, 2 \mathrm{H}, \mathrm{ArH}), 8.28$ (d, $J=8.0 \mathrm{~Hz}, 2 \mathrm{H}, \mathrm{ArH}) ;{ }^{13} \mathrm{C}$ NMR $\left(100 \mathrm{MHz}\right.$, DMSO- $\left.d_{6}\right) \delta$ : $201.2(\mathrm{C}-7), 173.1(\mathrm{C}-24), 166.5(\mathrm{PhC}=\mathrm{O}), 153.2(\mathrm{Ph})$, $136.5(\mathrm{Ph}), 131.1$ (Ph, 2C), 124,3 (Ph, 2C), 144.7 (C-5), 126.8 (C-7), 74.1 (C-3), 54.1 (C-17), 51.1 (C-25), 48.2 (C-9), 21.1 (C-10), 19.3 (C-21), 13.3 (C-19, C-18); HRMS (EI) calcd for $\mathrm{C}_{32} \mathrm{H}_{41} \mathrm{O}_{7} \mathrm{~N}: 551.2883$, found 551.2878.

\section{$1.53 \alpha$-对硝基苯甲酰基- $7 \beta$-羟基- $\Delta^{5}$-胆烷酸甲酯(7)的} 合成

在 $25 \mathrm{~mL}$ 的圆底瓶中加入化合物 $6(1 \mathrm{mmol})$, $\mathrm{NaBH}_{4}(1.2 \mathrm{mmol}), \mathrm{CeCl}_{3}(1.2 \mathrm{mmol})$ 和 $10 \mathrm{~mL}$ 甲醇, 室 温条件下搅拌, TLC 跟踪反应, 反应完全时, 减压除去 大部分的有机溶剂, 再向体系中加入 $50 \mathrm{~mL}$ 水, 乙酸乙 酯萃取 $(25 \mathrm{~mL} \times 3)$, 有机相用饱和 $\mathrm{NaCl}$ 溶液洗涤, 无 水 $\mathrm{Na}_{2} \mathrm{SO}_{4}$ 干燥, 浓缩得粗品. 快速硅胶柱层析[洗脱剂: $V$ (石油醚) $: V$ (乙酸乙酯) $=8: 2$ ]得白色固体 $70.38 \mathrm{~g}$, 产率为 $68 \%$. m.p. $174 \sim 177{ }^{\circ} \mathrm{C} ;{ }^{1} \mathrm{H}$ NMR $(400 \mathrm{MHz}$, $\left.\mathrm{CDCl}_{3}\right) \delta: 0.69\left(\mathrm{~s}, 3 \mathrm{H}, 18-\mathrm{CH}_{3}\right), 0.93(\mathrm{~d}, J=6.2 \mathrm{~Hz}, 3 \mathrm{H}$, $\left.21-\mathrm{CH}_{3}\right), 0.97 \sim 2.63(\mathrm{~m}, 23 \mathrm{H}), 1.16\left(\mathrm{~s}, 3 \mathrm{H}, 19-\mathrm{CH}_{3}\right), 3.60$ (s, 3H, $\mathrm{COOCH}_{3}$ ), 3.93 (brs, 1H, 7-H), 4.64 4.66 (brm, $1 \mathrm{H}, 3-\mathrm{H}$ ), 5.82 (brs, $1 \mathrm{H}, 6-\mathrm{H}), 8.12$ (d, $J=7.9 \mathrm{~Hz}, 2 \mathrm{H}$, ArH), 8.27 (d, $J=7.9 \mathrm{~Hz}, 2 \mathrm{H}, \mathrm{ArH}) ;{ }^{13} \mathrm{C}$ NMR $(100 \mathrm{MHz}$, DMSO- $\left.d_{6}\right) \delta: 173.1(\mathrm{C}-24), 165.9(\mathrm{PhC}=\mathrm{O}), 152.7(\mathrm{Ph})$, $136.3(\mathrm{Ph}), 131.5(\mathrm{Ph}, 2 \mathrm{C}), 124.3(\mathrm{Ph}, 2 \mathrm{C}), 145.1$ (C-5), 73.7 (C-3), 65.4 (C-7), 54.9 (C-17), 50.1 (C-25), 48.2 (C-9), 21.5 (C-10), 19.0 (C-21), 13.1 (C-19, C-18); HRMS (EI) calcd for $\mathrm{C}_{32} \mathrm{H}_{43} \mathrm{O}_{7} \mathrm{~N}$ : 553.3039, found 553.3042.

\section{$1.63 \alpha$-对硝基苯甲酰基- $7 \beta$-羟基-胆烷酸甲酯(8)的合 成}

化合物 $7(1 \mathrm{mmol})$ 溶解于 $15 \mathrm{~mL}$ 甲醇中, 再加入催 化剂量的 $5 \% \mathrm{Pd} / \mathrm{C}$, 用带有 $\mathrm{H}_{2}$ 气球的三通活塞, 在减压 的条件下, 来回置换反应体系中的空气 3 次, 使反应体 系尽量在 $\mathrm{H}_{2}$ 氛围中, 室温条件下剧烈摚拌, TLC 跟踪反 应，反应完全时，过滤，滤饼用甲醇洗 3 次，滤液减压除 去大部分甲醇, 再加入 $30 \mathrm{~mL}$ 水, 乙酸乙酯萃取 $(25$ $\mathrm{mL} \times 3$ ) 有机相用饱和 $\mathrm{NaCl}$ 溶液洗涤, 无水 $\mathrm{Na}_{2} \mathrm{SO}_{4}$ 干 燥, 浓缩得粗品. 快速硅胶柱层析[洗脱剂: $V$ (石油醚)： $V($ 乙酸乙酯 $)=8: 2$ ]得固体 $80.47 \mathrm{~g}$, 产率为 $85 \%$. m.p. $183 \sim 187{ }^{\circ} \mathrm{C} ;{ }^{1} \mathrm{H}$ NMR $\left(400 \mathrm{MHz}, \mathrm{CDCl}_{3}\right) \delta: 0.69$ (s, 3H, $\left.18-\mathrm{CH}_{3}\right), 0.93\left(\mathrm{~d}, J=6.2 \mathrm{~Hz}, 3 \mathrm{H}, 21-\mathrm{CH}_{3}\right), 1.01 \sim 2.60(\mathrm{~m}$, $26 \mathrm{H}$ ), 1.16 (s, 3H, 19- $\mathrm{CH}_{3}$ ), 3.60 (s, 3H, $\mathrm{COOCH}_{3}$ ), 3.90 $3.92(\mathrm{~m}, 1 \mathrm{H}, 7 \alpha-\mathrm{H}), 5.25$ (brs, 1H, H-6), 5.34 (brs, $1 \mathrm{H}$, 3-H), 8.12 (d, $J=7.9 \mathrm{~Hz}, 2 \mathrm{H}, \mathrm{ArH}), 8.27$ (d, $J=7.9 \mathrm{~Hz}$, $2 \mathrm{H}, \mathrm{ArH}) ;{ }^{13} \mathrm{C}$ NMR (100 MHz, DMSO- $\left.d_{6}\right) \delta: 174.0$ $(\mathrm{C}-24), 165.4(\mathrm{PhC}=\mathrm{O}), 137.1(\mathrm{Ph}), 132.3(\mathrm{Ph}, 2 \mathrm{C}), 125.5$ (Ph, 2C), 74.2 (C-3), 56.9 (C-17), 51.1 (C-25), 49.2 (C-9), 22.3 (C-10), 20.1 (C-21), 13.5 (C-19, C-18); HRMS (EI) calcd for $\mathrm{C}_{32} \mathrm{H}_{45} \mathrm{O}_{7} \mathrm{~N}$ : 555.3196, found 555.3192.

\section{7 异熊去氧胆酸(9)的合成}

化合物 $81 \mathrm{~g}(1.8 \mathrm{mmol})$ 溶于 $10 \mathrm{~mL}$ 甲醇中, 再加入 $10 \mathrm{~mL} 4 \% \mathrm{NaOH}(0.1 \mathrm{~mol} / \mathrm{L})$ 溶液, 室温反应 $20 \mathrm{~min}$ 后, 加入 $5 \% \mathrm{HCl}(0.13 \mathrm{~mol} / \mathrm{L})$ 溶液调到 $\mathrm{pH} 3$, 减压除去甲醇 后，乙酸乙酯萃取 $(25 \mathrm{~mL} \times 3)$ 有机相用饱和 $\mathrm{NaCl}$ 溶液 洗涤, 无水 $\mathrm{Na}_{2} \mathrm{SO}_{4}$ 干燥, 浓缩得粗品. 快速硅胶柱层析 [洗脱剂: $V$ (石油醚)： $V$ (乙酸乙酯) $=6: 4$ ] 得到无色针状 固体 $90.66 \mathrm{~g}$, 产率为 $93 \%$. m.p. $243 \sim 245^{\circ} \mathrm{C}$ (文献值 ${ }^{[1]}$ : m.p. $\left.243.5 \sim 245{ }^{\circ} \mathrm{C}\right) ;{ }^{1} \mathrm{H}$ NMR (400 MHz, $80 \% \mathrm{CDCl}_{3}+$ $20 \%$ DMSO- $\left.d_{6}\right) \delta: 0.68\left(\mathrm{~s}, 3 \mathrm{H}, 18-\mathrm{CH}_{3}\right), 0.79(\mathrm{~s}, 3 \mathrm{H}$, $\left.19-\mathrm{CH}_{3}\right), 0.93(\mathrm{~d}, J=5.6 \mathrm{~Hz}, 3 \mathrm{H}, \mathrm{H}-21), 0.97 \sim 2.51(\mathrm{~m}$, 26H), $3.47 \sim 3.49$ (brm, 1H, H-7), $3.97 \sim 3.99(\mathrm{~m}, 1 \mathrm{H}$, $\mathrm{H}-3) ;{ }^{13} \mathrm{C}$ NMR (100 MHz, DMSO- $\left.d_{6}\right) \delta: 175.3(\mathrm{COOH})$, 70.2 (C-3), 69.8 (C-7), 56.3 (C-17), 51.1 (C-9), 18.7 (C-21), 12.5 (C-18, C-19); IR (KBr) $v_{\max }: 3410(\mathrm{O}-\mathrm{H})$, 
$1776(\mathrm{C}=\mathrm{O}), 1034(\mathrm{C}-\mathrm{O}) \mathrm{cm}^{-1}$; HRMS (EI) calcd for $\mathrm{C}_{24} \mathrm{H}_{40} \mathrm{O}_{4}: 392.2927$, found 392.2929 .

\section{2 结果与讨论}

猪去氧胆酸含量占猪胆汁酸的 $40 \%$, 是我国丰富的 甾体资源. 从猪去氧胆酸合成异熊去氧胆酸的关键步骤 是使 $\mathrm{A} / \mathrm{B}$ 环顺式变为反式稠合, 同时使 $6 \alpha$-羟基移位成 $7 \beta$-羊基. 我们通过 9 步反应, 以总收率 $25 \%$ 完成了目标 产物的合成, 对每一步反应条件进行了优化. 首先猪去 氧胆酸经甲酯化得到 1,1 在室温条件下与对甲苯磺酰氯 反应生成二磺酰化的产物 2,2 在 $\mathrm{CH}_{3} \mathrm{CO}_{2} \mathrm{~K}-\mathrm{KOH}$ 的 DMF- $\mathrm{H}_{2} \mathrm{O}$ 体系中回流后, 再经乙酰化得到乙酰化的产 物 3.3 经烯丙位氧化生成 $\alpha, \beta$-不饱和酮类化合物 $\mathbf{4}$, 传 统的烯丙位直接氧化生成 $\alpha, \beta$-不饱和醛酮的方法中, 最 常用的试剂有 $\mathrm{CrO}_{3}-3,5$-二甲基吡唑, PCC, PDC, 重铬酸 钠、重铬酸钠-冰乙酸等, 这些方法中, 铬试剂用量都很 大, 有时甚至达到 120 倍摩尔量, 因而使其后处理十分 麻烦, 不仅铬试剂对产物的吸附较大, 而且高价铬的毒 性, 对环境造成严重的污染 ${ }^{[8]}$. 我们对 $\mathrm{CrO}_{3}$-吡啶络合物 氧化体系进行了改进, 在反应体系中引入过氧叔丁醇 (TBHP) 进行辅助氧化, 可大大减少三氧化铬的使用量, 减少了对环境的不利影响.

化合物 4 在 $\mathrm{K}_{2} \mathrm{CO}_{3} / \mathrm{KHCO}_{3}$ 的弱碱性水溶液中水解 脱去乙酰基生成醇 5,5 在偶氮二羧酸二乙酯(DIAD)和 三苯基膦存在下经过 Mitsunobu 构型反转反应得到化合 物 6, 该步反应条件温和, 产率高并发生 $\mathrm{S}_{\mathrm{N}} 2$ 构型翻 转 ${ }^{[9,10]}$. 对 Mitsunobu 反应来说, 投料顺序和反应温度控 制很重要, 首先应当将对硝基苯甲酸、底物醇、三苯基 膦溶解于适当溶剂如四氢呋喃或乙醚中, 冰水浴冷至 0 ${ }^{\circ} \mathrm{C}$, 将 DIAD 缓慢滴入, 然后在室温条件下摚拌反应 24 $\mathrm{h}$, 在这种反应条件下, 反应的收率较高. 若直接将 DIAD 与其他底物一起投入或滴加 DIAD 在室温下进行, 反应的副产物会增加, 收率明显下降. $\alpha, \beta$-不饱和酮 $\mathbf{6}$ 在 Luche 条件下采用 $\mathrm{CeCl}_{3} / \mathrm{NaBH}_{4}$ 还原体系 ${ }^{[1]}$ 经立体选择 性还原为相应的烯丙醇 7, 产物经 ${ }^{1} \mathrm{H}$ NMR 分析并与文 献 ${ }^{[12]}$ 对比证明其 7 位羟基为 $\beta$ 构型 $[\delta 3.90(7 \alpha-\mathrm{H})$ 是多重 峰, $\delta 5.25(6-\mathrm{H})$ 是宽单峰], 含量 $\geqslant 90 \%$. 化合物 7 用 $5 \%$ $\mathrm{Pd} / \mathrm{C}$ 催化氢化, 我们曾试图获得 $5 \beta$ 构型的化合物, 它可
进一步水解得熊去氧胆酸, 但只得到 $5 \alpha$ 构型的化合物 $\boldsymbol{8}^{[7]}, \mathbf{8}$ 经 $4 \% \mathrm{NaOH}$ 溶液水解得到异熊去氧胆酸 9 . 所有 合成化合物的结构已通过 ${ }^{1} \mathrm{H} N M R,{ }^{13} \mathrm{C}$ NMR, MS 和 IR 等波谱方法进行了确认.

\section{3 结论}

从猪去氧胆酸出发经 9 步合成异熊去氧胆酸(总产 率为 $25 \%$ ), 类似方法未见文献报道. 该合成方法有如下 优点: (1)合成路线新颖, 猪去氧胆酸来源丰富, 原料易 得. (2)立体选择性好, 二步关键步骤中, Mistunobu 构型 转化可达 $100 \%$ 的构型翻转, Luche 还原得到 $3 \beta$-差弪基化 物含量 $90 \%$ 以上. (3)反应条件温和, 所采用的合成路线 不需要高温高压反应条件或低温操作，易于实现规模化 合成, 因而具有较高的应用价值.

\section{References}

[1] Iida, T.; Momose, T.; Nambara, T.; Chang, F. C. Chem. Pharm. Bull. 1986, 34, 1929.

[2] Alvarez, M.; Jover, A.; Carrazana, J.; Meijide, F.; Soto, V. H.; Tato, J. V. Steroids 2007, 72, 535.

[3] Tohma, M.; Mahara, R.; Takeshita, H.; Kurosawa, T. Steroids 1986, 48(5 6), 331 .

[4] Goto, T. Proc. Jpn. Acad. 1955, 31, 466.

[5] Shen, J. M.; Zhou, X. D.; Zhou, W. S. Acta Chim. Sinica 2006, 64, 1513 (in Chinese).

(沈季铭, 周向东, 周维善, 化学学报, 2006, 64, 1513.)

[6] Iida, T.; Kakiyama, G.; Hibiya, Y.; Miyata, S.; Inoue ,T.; Ohno, K.; Goto, T.; Mano, N.; Goto, J.; Nambara, T.; Hofmann, A. F. Steroids 2006, 71,18

[7] Wang, Z. Q.; Que, H. Q.; Jiang, L. Z.; Zhou W. S. Chin. J. Org. Chem. 1989, 9, 83 (in Chinese). (王锤麒, 洅浩泉, 姜立中, 周维善, 有机化学, 1989,9,83.)

[8] Ge, Y. C.; Yang, Q. X.. J. Guizhou Normal Univ. (Nat. Sci.) 2009, 27,119 (in Chinese).

(葛永昌, 杨庆雄, 贵州师范大学学报 (自然科学版), 2009, 27, 119.)

[9] Xu, X. L.; Cheng, G. H., Li, X. J. Chin. J. Org. Chem. 2012, 32, 1024 (in Chinese). (许孝良, 程国华, 李小军, 有机化学, 2012, 32, 1024.)

[10] Wang, Q. A.; Luo, J. X.; Liao, T. G. Chem. J. Chin. Univ. 2004, 25, 1069 (in Chinese). (汪秋安, 罗俊霞, 廖头根, 高等学校化学学报, 2004, 25, 1069.)

[11] Gimed, A. L.; Luche, J. L. J. Am. Chem. Soc. 1981, 103, 5454

[12] Zheng, D.; Guan, Y. Y.; Chen, X. Z.; Xu, Y. P.; Chen, X. G.; Lei, P. S. Bioorg. Med. Chem. Lett. 2011, 21, 3257. 